\title{
Instanton Particles and Monopole Strings in 5D SU(2) Supersymmetric Yang-Mills Theory
}

\author{
Pietro Longhiఠ* \\ Institute for Theoretical Physics, ETH Zurich, 8093 Zurich, Switzerland
}

(Received 16 January 2021; accepted 28 April 2021; published 25 May 2021)

\begin{abstract}
We provide a closed-form expression for the motivic Kontsevich-Soibelman invariant for $M$ theory in the background of the toric Calabi-Yau threefold $K_{\mathrm{F}_{0}}$. This encodes the refined Bogomol'nyi-PrasadSommerfield spectrum of $\mathrm{SU}(2) 5 \mathrm{D} \mathcal{N}=1$ Yang-Mills theory on $S^{1} \times \mathbb{R}^{4}$, corresponding to rank-zero Donaldson-Thomas invariants for $K_{\mathrm{F}_{0}}$, anywhere on the Coulomb branch.
\end{abstract}

DOI: 10.1103/PhysRevLett.126.211601

Introduction and main result.-Quantum field theory (QFT) enables quantitative descriptions of natural phenomena with remarkable flexibility, ranging from microscopic to cosmological scales. While the importance of this framework as a driver of discovery is well established, QFT still harbors unsolved mysteries of central importance. Understanding strongly coupled dynamics certainly deserves a place among the most pressing ones, due to its omnipresence in nature: for example, quark confinement underlies the existence of stable nucleons, the basic building blocks of atoms and all ordinary matter.

Many of these questions can be approached fruitfully within the theoretical laboratory of gauge theories with supersymmetry (SUSY). This laboratory is both abundant in examples and rich in tools to address a variety of questions on strongly coupled phenomena, including confinement [1]. The hallmark of SUSY gauge theories is the existence of Bogomol'nyi-Prasad-Sommerfield (BPS, or protected) sectors, whose properties offer a window into strong coupling dynamics, well beyond the reach of conventional perturbation theory based on Lagrangian or Hamiltonian descriptions. The study of BPS states has been appreciated as a promising approach to a variety of deep questions, ranging from confinement to microscopic models of black hole entropy.

In this Letter we provide an exact description of the BPS spectrum of minimally supersymmetric five-dimensional (5D) SU(2) Yang-Mills gauge theory. While this theory is superficially nonrenormalizable, it is known to flow to a nontrivial fixed point with enhanced global symmetry [2]. To our knowledge, this is the first complete description of the BPS spectrum of a $5 \mathrm{D} \mathcal{N}=1$ gauge theory. The first

Published by the American Physical Society under the terms of the Creative Commons Attribution 4.0 International license. Further distribution of this work must maintain attribution to the author(s) and the published article's title, journal citation, and DOI. Funded by SCOAP ${ }^{3}$. important novelty in our approach is that, instead of focusing on the study of single BPS states, we obtain an exact characterization of the full spectrum by computing directly the wall-crossing invariant of Kontsevich and Soibelman [3]. The second novelty in our approach is that we obtain the invariant by combining partial information obtained at different choices of stability conditions.

BPS states of 5D theories with eight supercharges are also interesting from a mathematical viewpoint because their BPS indices correspond to rank-zero Donaldson-Thomas invariants of certain Calabi-Yau threefolds. The theory considered here corresponds to the canonical bundle of the Hirzebruch surface $\mathbb{F}_{0}[2,4,5]$. Enumerative invariants of geometries with compact four cycles are notoriously challenging to compute. Exhaustive results are scarce, except for recent developments for local $\mathbb{P}^{2}$ based on scattering diagrams [6] and results based on conjectures for attractor invariants [7].

A given theory typically has not one but many different BPS spectra, corresponding to different chambers of moduli space and related by wall crossing [3,8-10]. The wall-crossing invariant of Kontsevich and Soibelman, also known as the (motivic) spectrum generator, encodes all possible spectra. A standard way to compute this invariant, henceforth denoted by $\mathbb{U}$, requires knowing the full BPS spectrum at some (any) point in Kähler moduli space. When this information is unavailable, as in our case, the only way to obtain the invariant is to look for alternative definitions. Following [11], one may hope to compute $\mathbb{U}$ at the Roman locus of [12], based on computations with spectral or exponential networks [13,14]. Unfortunately, the existence of Roman loci is poorly understood at the moment, both for $4 \mathrm{D} \mathcal{N}=2$ and 5D $\mathcal{N}=1$ theories. In this Letter we introduce a new approach to computing $\mathbb{U}$, based on leveraging information about BPS states at different points in moduli space studied in $[15,16]$. Details of this will be explained below.

Fixing a choice of Kähler moduli determines a unique BPS spectrum, with BPS states of charge $\gamma \in \Gamma$ 
characterized by a central charge $Z_{\gamma} \in \operatorname{Hom}(\Gamma, \mathbb{C})$. Here $\Gamma \simeq \mathbb{Z}^{4}$ is a lattice of charges endowed with a skewsymmetric bilinear form $\langle\cdot, \cdot\rangle$, the Dirac-SchwingerZwanziger pairing. CPT symmetry implies that if the spectrum features a state with charge $\gamma$, there is a corresponding state with charge $-\gamma$. We henceforth focus on studying the half of the spectrum with $-\pi / 2 \leq \operatorname{Arg} Z_{\gamma}<\pi / 2$, whose charges $\gamma$ are positiveinteger linear combinations of four basic charges $\gamma_{1}, \ldots, \gamma_{4}$ fixed by the choice of half-plane. In this, note $\gamma_{i}$ correspond to exceptional sheaves on $K_{\mathbb{E}_{0}}$, or fractional D-branes in IIA string theory:

\begin{tabular}{c|c|c|c}
$\gamma_{1}$ & $\gamma_{2}$ & $\gamma_{3}$ & $\gamma_{4}$ \\
\hline $\mathcal{O}(0,0)$ & $\mathcal{O}(1,0)$ & $\mathcal{O}(1,1)$ & $\mathcal{O}(2,1)$ \\
\hline$D 4$ & $D 2_{f}-\overline{D 4}$ & $D 0-D 2_{b}-\overline{D 2}_{f}-\overline{D 4}$ & $\overline{D 2}_{b}-D 4$
\end{tabular}

where $D 2_{b}$ (resp. $D 2_{f}$ ) denotes a D2-brane wrapping the base (resp. fiber) $\mathbb{P}^{1}$ in $\mathbb{F}_{0}$ and overlines denote antibranes. The pairing is $\left\langle\gamma_{i}, \gamma_{i+1}\right\rangle=-2$ with $i \in \mathbb{Z} / 4 \mathbb{Z}$; see [16].

The BPS spectrum is encoded by the BPS index $\Omega(\gamma) \in \mathbb{Z}$. By the no-exotics phenomenon, $|\Omega(\gamma)|$ actually counts states [17-19]. The protected spin character (PSC) $\Omega(\gamma ; y)$ is a Laurent polynomial with integer coefficients, which refines the BPS index by encoding information about the spin of BPS states. The relation between the two is $\Omega(\gamma)=\Omega(\gamma ; y=-1)$. Mathematically the BPS index should coincide with numerical rank-zero DonaldsonThomas invariants, while the PSC should correspond to their motivic version.

Having spelled out necessary conventions, we can state the main result for the motivic wall-crossing invariant

$$
\begin{aligned}
\mathbb{U}= & \prod_{k \geq 0} \Phi\left(\hat{Y}_{\gamma_{1}+k\left(\gamma_{1}+\gamma_{2}\right)}\right) \Phi\left(\hat{Y}_{\gamma_{3}+k\left(\gamma_{3}+\gamma_{4}\right)}\right) \\
& \times \prod_{n \geq 1} \Phi\left[(-y)^{-1} \hat{Y}_{n\left(\gamma_{1}+\gamma_{2}+\gamma_{3}+\gamma_{4}\right)}\right]^{-1} \Phi\left[(-y) \hat{Y}_{n\left(\gamma_{1}+\gamma_{2}+\gamma_{3}+\gamma_{4}\right)}\right]^{-2} \Phi\left[(-y)^{3} \hat{Y}_{n\left(\gamma_{1}+\gamma_{2}+\gamma_{3}+\gamma_{4}\right)}\right]^{-1} \\
& \times \prod_{k \geq 0} \Phi\left[(-y)^{-1} \hat{Y}_{\gamma_{1}+\gamma_{2}+k\left(\gamma_{1}+\gamma_{2}+\gamma_{3}+\gamma_{4}\right)}\right]^{-1} \Phi\left[(-y) \hat{Y}_{\gamma_{1}+\gamma_{2}+k\left(\gamma_{1}+\gamma_{2}+\gamma_{3}+\gamma_{4}\right)}\right]^{-1} \\
& \times \prod_{k \geq 0} \Phi\left[(-y)^{-1} \hat{Y}_{\gamma_{3}+\gamma_{4}+k\left(\gamma_{1}+\gamma_{2}+\gamma_{3}+\gamma_{4}\right)}\right]^{-1} \Phi\left[(-y) \hat{Y}_{\gamma_{3}+\gamma_{4}+k\left(\gamma_{1}+\gamma_{2}+\gamma_{3}+\gamma_{4}\right)}\right]^{-1} \\
& \times \prod_{k \geq 0}^{\searrow} \Phi\left(\hat{Y}_{\gamma_{2}+k\left(\gamma_{1}+\gamma_{2}\right)}\right) \Phi\left(\hat{Y}_{\gamma_{4}+k\left(\gamma_{3}+\gamma_{4}\right)}\right),
\end{aligned}
$$

where $\Phi(\xi)=\prod_{s \geq 0}\left(1+y^{2 s+1} \xi\right)^{-1}$ is a variant of the quantum dilogarithm function, $\hat{Y}_{\gamma}$ are quantum-torus variables obeying $\hat{Y}_{\gamma} \hat{Y}_{\gamma^{\prime}}=y^{\left\langle\gamma, \gamma^{\prime}\right\rangle} \hat{Y}_{\gamma+\gamma^{\prime}}$, and $\nearrow$ (respectively $\searrow$ ) denotes increasing (decreasing) values of $k$ to the right. An important clarification is now in order: we did not find a point in moduli space where central charges have phases arranged in this configuration and where the spectrum is so simple: this is an expression for $\mathbb{U}$, not for the BPS spectrum. Nonetheless, there exists a virtual configuration of central charges $Z_{\gamma_{i}}$ such that the factors in (2) are ordered according to the phase of $Z_{\gamma}$.

Derivation.-We now explain how (2) is derived. Suppose we fix a generic choice of moduli, away from walls of marginal stability. Given any angular sector $\measuredangle \subset \mathbb{C}$, we may define $\mathbb{U}(\measuredangle)$ as the phase-ordered product of $\Phi\left((-y)^{m} \hat{Y}_{\gamma}\right)^{a_{m}(\gamma)}$ for any $\gamma$ with $Z_{\gamma} \in \measuredangle$. Here $a_{m}(\gamma) \in \mathbb{Z}$ are coefficients of the PSC, namely $\Omega(\gamma ; y)=\sum_{m \in \mathbb{Z}}(-y)^{m} a_{m}(\gamma)$. We split the half-plane $-\pi / 2 \leq \operatorname{Arg} Z<\pi / 2$ into three sectors: the positive real line $\mathbb{R}^{+}$, the sector $\measuredangle^{+}$corresponding to $0<\operatorname{Arg} Z<\pi / 2$, and the sector $\measuredangle^{-}$corresponding to $-\pi / 2 \leq \operatorname{Arg} Z<0$. This defines a decomposition of the wall-crossing invariant $\mathbb{U}=\mathbb{U}\left(\measuredangle^{+}\right) \cdot \mathbb{U}\left(\mathbb{R}^{+}\right) \cdot \mathbb{U}\left(\measuredangle^{-}\right)$.

The wall-crossing formula of Kontsevich and Soibelman asserts that $\mathbb{U}(\varangle)$ is invariant under changes of stability conditions (i.e., central charges [20,21]), as long as no BPS rays enter or exit the sector $\measuredangle$. By a BPS ray we mean any locus $Z_{\gamma} \mathbb{R}^{+} \subset \mathbb{C}$ such that $\Omega(\gamma ; y) \neq 0$. We will take advantage of this invariance property to compute different factors of $\mathbb{U}$ at different points in the moduli space of stability conditions: in particular we will compute $\mathbb{U}\left(\measuredangle^{ \pm}\right)$ for a certain configuration of central charges and $\mathbb{U}\left(\mathbb{R}^{+}\right)$for a different one. All we need to ensure is that these configurations of central charges are connected by a variation of central charges that never causes a BPS ray to cross the boundaries of sectors $\measuredangle^{ \pm}$.

A path in the moduli space of stability conditions.-We consider the mirror geometry of $K_{\mathbb{F}_{0}}$, described by a conic 
bundle over the algebraic curve $1-Q_{b}\left(x+x^{-1}\right)+$ $Q_{f}\left(y+y^{-1}\right)=0$ in $\mathbb{C}_{x}^{*} \times \mathbb{C}_{y}^{*}$. The four basic charges are identified with homology cycles on this curve; see [16] for a detailed description. Central charges are determined by periods of the 1 form $\lambda=(2 \pi)^{-1} \log y(d x / x)$ and can be evaluated numerically. For $Q_{b}=-1, Q_{f}=2$ one finds that $\pi / 2>\operatorname{Arg} Z_{\gamma_{3}}>\operatorname{Arg} Z_{\gamma_{1}}>0$ and $\operatorname{Arg} Z_{\gamma_{2}}=$ $\operatorname{Arg} Z_{\gamma_{4}}=-\pi / 2$, and in particular

$$
Z_{\gamma_{1}+\gamma_{2}}, \quad Z_{\gamma_{3}+\gamma_{4}} \in \mathbb{R}^{+} .
$$

Numerical evaluation yields $Z_{\gamma_{1}} \approx 2.59433+0.349113 i$, $Z_{\gamma_{2}} \approx-0.349113 i, \quad Z_{\gamma_{3}} \approx 3.68886+1.03718 i, \quad Z_{\gamma_{4}} \approx$ $-1.03718 i$. The reality condition (3) can also be verified by plotting the exponential network at $\vartheta=0$, where both saddles of $\gamma_{1}+\gamma_{2}$ and of $\gamma_{3}+\gamma_{4}$ appear; see Fig. 1. The ray $\mathbb{R}^{+}$contains central charges of all states in the span of $\gamma_{1}+\gamma_{2}$ and $\gamma_{3}+\gamma_{4}$. Note that these are mutually local, i.e., $\left\langle\gamma_{1}+\gamma_{2}, \gamma_{3}+\gamma_{4}\right\rangle=0$ (the same holds for charges in their span); this ensures we are not on a wall of marginal stability. This is the starting point $t=0$ of the path in the moduli space of central charge configurations $\tilde{Z}_{\gamma_{1}}(t)=$ $(1-t) Z_{\gamma_{1}}+t Z_{\gamma_{3}}, \tilde{Z}_{\gamma_{2}}(t)=(1-t) Z_{\gamma_{2}}+t Z_{\gamma_{4}} \tilde{Z}_{\gamma_{3}}(t)=Z_{\gamma_{3}}$, $\tilde{Z}_{\gamma_{4}}(t)=Z_{\gamma_{4}}$. The path ends at $t=1$, where

$$
Z_{\gamma_{1}}^{\prime}=Z_{\gamma_{3}}^{\prime} \in 4^{+} \quad Z_{\gamma_{2}}^{\prime}=Z_{\gamma_{4}}^{\prime} \in{\chi^{-}}^{-}
$$

The path has the crucial property that $\tilde{Z}_{\gamma_{1}}(t)+\tilde{Z}_{\gamma_{2}}(t)$ is real and positive for all $t \in[0,1]$, thanks to (3). The configuration of central charges for $t=0$ is realized at a point in the moduli space of the (mirror) geometry, and we refer to it as a physical stability condition. On the other hand, the configurations $\tilde{Z}(t)$ for $t>0$ are not necessarily realized in moduli space, and we refer to them as virtual stability conditions. Configurations analogous to $t=0$ and $t=1$ were considered in [16] and [15], respectively.

Computing $\mathbb{U}\left(\measuredangle^{ \pm}\right)$at $t=1$. - The virtual stability condition for $t=1$ was analyzed by Closset and Del Zotto [15], whose approach we review. The BPS spectrum admits a description in terms of a BPS quiver with four nodes [22] labeled by $\gamma_{i}$; our conventions are adapted to [16]. For the choice of half-plane considered in this note, the structure of the quiver is cyclic, with two arrows from the $i$ th node to

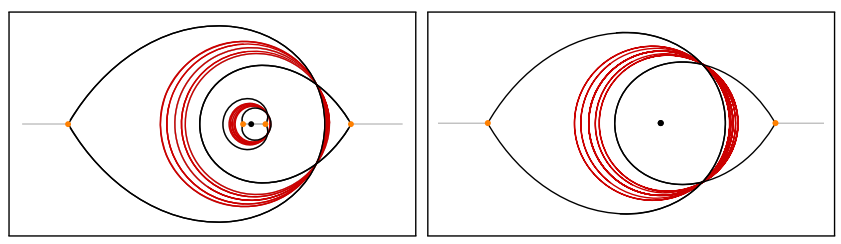

FIG. 1. Left: exponential network of local $\mathbb{F}_{0}$ at $\vartheta=0$ for $Q_{b}=-1, Q_{f}=2$. Right: exponential BPS graph for $\mathcal{O}(0) \oplus$ $\mathcal{O}(-2) \rightarrow \mathbb{P}^{1}$ in quadratic choice of framing. the $i+1$ th node. Tilting the choice of half-plane clockwise induces a periodic sequence of mutations, respectively on nodes $1,2,3,4$. This leads, by the mutation method of [23], to an infinite tower of hypermultiplets with charges $\gamma_{1}+k\left(\gamma_{1}+\gamma_{2}\right)$ and $\gamma_{3}+k\left(\gamma_{3}+\gamma_{4}\right)$ for $k \geq 0$. Similarly, counterclockwise rotations of the half-plane lead to a second tower of BPS hypermultiplets with charges $\gamma_{2}+k\left(\gamma_{1}+\gamma_{2}\right)$ and $\gamma_{4}+k\left(\gamma_{3}+\gamma_{4}\right)$. Note that the central charges of these towers both asymptote to $\mathbb{R}^{+}$, but from opposite sides; hence there is a single "accumulation" ray along $\mathbb{R}^{+}$.

Since the clockwise (resp. counterclockwise) tilting of the half-plane covers the whole angular sector $4^{+}$ (resp. $\left.\measuredangle^{-}\right)$, we deduce that $\mathbb{U}\left(\measuredangle^{ \pm}\right)$coincide respectively with the first and last line in (2). One should worry that $\operatorname{Arg} Z_{\gamma_{1}+k\left(\gamma_{1}+\gamma_{2}\right)}^{\prime}=\operatorname{Arg} Z_{\gamma_{3}+k\left(\gamma_{3}+\gamma_{4}\right)}^{\prime}$ may cause ordering ambiguities in the above formulas. But since $\left\langle\gamma_{1}+k\left(\gamma_{1}+\right.\right.$ $\left.\left.\gamma_{2}\right), \gamma_{3}+k^{\prime}\left(\gamma_{3}+\gamma_{4}\right)\right\rangle=0$ and $\left\langle\gamma_{2}+k\left(\gamma_{1}+\gamma_{2}\right), \gamma_{4}+\right.$ $\left.k^{\prime}\left(\gamma_{3}+\gamma_{4}\right)\right\rangle=0$ when $k=k^{\prime}$, the corresponding factors commute.

As we move from $t=1$ to the physical stability condition $t=0$, the BPS rays that contribute to $\mathbb{U}\left(\varangle^{ \pm}\right)$begin to move within $\measuredangle^{ \pm}$; however, they never exit these sectors. As BPS rays move around within $\measuredangle^{ \pm}$they may cross each other and generate new BPS rays by wall crossing. Any new rays generated in this way must lie in the cone of the two BPS rays that generated them, ensuring that even these descendants (and their own descendants) must be confined within one of $4^{ \pm}$as well. Furthermore, any BPS rays within $\mathbb{R}^{+}$at $t=1$ are confined there also for $0 \leq t<1$, never crossing into $\measuredangle^{ \pm}$. A direct way to see this is to notice that the quiver has a $\mathbb{Z}_{4}$ symmetry, which must be inherited by $\mathbb{U}[11]$. Enforcing this, with $\mathbb{U}\left(\measuredangle^{ \pm}\right)$as determined here, fixes $\mathbb{U}\left(\mathbb{R}^{+}\right)$ and establishes that it only contains contributions from BPS rays that remain in $\mathbb{R}^{+}$for all $t$. These facts imply that $\mathbb{U}\left(\measuredangle^{ \pm}\right), \mathbb{U}\left(\mathbb{R}^{+}\right)$obtained for the virtual stability condition at $t=1$ must coincide with their counterparts for the physical stability conditions at $t=0$.

Computing $\mathbb{U}\left(\mathbb{R}^{+}\right)$at $t=0$.-What is left out by the above analysis is to determine the part of $\mathbb{U}$ corresponding to the accumulation ray. This can actually be obtained quite easily, by plotting the exponential network at $Q_{b}=-1, Q_{f}=2$ (corresponding to $t=0$ ) for $\vartheta=0$; see Fig. 1.

To determine the BPS spectrum encoded by the saddle one may use the machinery of $[14,16,24,25]$. But in this case one can take a shortcut. Note that saddles are divided into two disconnected parts. Each set has the same topology as the exponential BPS graph of $\mathcal{O}(0) \oplus \mathcal{O}(-2) \rightarrow \mathbb{P}^{1}$, shown in Fig. 1. More precisely, this is the exponential network at $\vartheta=0$ for $1+y+x y+$ $Q y^{2}=0$ with $Q=6$. It is equivalent (up to framing, which has no effect on BPS states) to the curve studied in [25], Eq. (4.3). Recall that (exponential) BPS graphs 
encode the whole BPS spectrum of a theory [11]. In the case of the half-geometry, the spectrum is known to consist of $\Omega(n D 0)=-2$ for $n \geq 1, \Omega(D 2-k D 0)=-1$ for $k \geq 0$, and $\Omega(\overline{D 2}-k D 0)=-1$ for $k \geq 1$ plus $C P T$ conjugates; see, e.g., [25], Eq. (4.34).

To translate this result into the BPS states in $\mathbb{U}\left(\mathbb{R}^{+}\right)$we simply have to identify $D 2$ with $D 2_{f}$ (cf. [16], Sec. V). Noting that $\gamma_{1}+\gamma_{2}=D 2_{f}$ and $\gamma_{3}+\gamma_{4}=D 0-\overline{D 2}_{f}$, and taking into account that the left frame in Fig. 1 contains two disconnected copies of the saddle in the right frame, we arrive at the following BPS indices:

$$
\begin{array}{rl}
\Omega\left(n\left(\gamma_{1}+\gamma_{2}+\gamma_{3}+\gamma_{4}\right)\right)=-4 & n \geq 1, \\
\Omega\left(\gamma_{1}+\gamma_{2}+k\left(\gamma_{1}+\gamma_{2}+\gamma_{3}+\gamma_{4}\right)\right)=-2 & k \geq 0, \\
\Omega\left(\gamma_{3}+\gamma_{4}+k\left(\gamma_{1}+\gamma_{2}+\gamma_{3}+\gamma_{4}\right)\right)=-2 & k \geq 0 .
\end{array}
$$

To promote BPS indices to PSCs, we note that states with $\Omega=-2$ are vector multiplets (seen, e.g., by the topology of their saddles; cf. [26]), whose PSC is $\Omega(\gamma ; y)=y+y^{-1}$. For the states with $\Omega=-4$ one should instead note that $\gamma_{D 0}=$ $\gamma_{1}+\gamma_{2}+\gamma_{3}+\gamma_{4}$ is the charge of a pure D0-brane. The motivic DT invariant of $n D 0$-branes in $K_{\mathbb{E}_{0}}$ was recently argued to be $\Omega(\gamma, y)=y^{-1}\left(1+y^{2}\right)^{2}$ in [7]. This completes the description of BPS states with real central charge, leading to $\mathbb{U}\left(\mathbb{R}^{+}\right)$given by the three central lines in (2) As discussed earlier, all charges appearing in this expression are mutually local, ensuring no ordering ambiguities.

Refined spectrum at a fiber-base symmetric point.-The mirror geometry of $K_{\mathbb{F}_{0}}$ has a symmetry under exchange of fiber and base moduli whenever $Q_{b}= \pm Q_{f}$. The point $Q_{b}=-1, Q_{f}=1$ was studied extensively in [16], where central charges were evaluated to be $Z_{\gamma_{1}} \approx 12.1717$, $Z_{\gamma_{2}} \approx 3.15831 i, Z_{\gamma_{3}} \approx 27.3067, Z_{\gamma_{4}} \approx-3.15831 i$. It is tedious but straightforward to compute the corresponding factorization of $\mathbb{U}$; see [11], Appendix E for an algorithm. Eventually we obtain the invariants listed in Table I, where $\left(n_{1}, n_{2}, n_{3}, n_{4}\right)$ is the shorthand for $\sum_{i=1}^{4} n_{i} \gamma_{i}$. The full spectrum is infinite; this list includes all states up to $|\gamma| \leq 7$. On specialization $y \rightarrow-1$, the spectrum in Table I recovers the unrefined spectrum obtained in [16] up to degree $|\gamma| \leq 6$ and predicts several new states for $|\gamma|>6$.

Discussion.-In this Letter we provided an exact expression for the motivic wall-crossing invariant of Kontsevich and Soibelman for the BPS spectrum of $M$ theory on $K_{\mathbb{F}_{0}}$. This operator encodes the spectrum of BPS states for any generic choice of Kähler moduli. In terms of gauge theory, $\mathbb{U}$ is the motivic spectrum generator for BPS monopole strings and instanton particles of 5D $\mathcal{N}=1 \mathrm{SU}(2)$ YangMills theory on $S^{1} \times \mathbb{R}^{4}$. From a geometric viewpoint, it encodes the spectrum of rank-zero (generalized) Donaldson-Thomas invariants for $K_{\mathbb{F}_{0}}$.

The derivation is based on data on BPS states at two different points in the moduli space of stability conditions,

\begin{tabular}{|c|c|}
\hline$\gamma$ & $\Omega(\gamma ; y)$ \\
\hline$(n, n, n, n)$ & $y^{3}+2 y+y^{-1}(n>0)$ \\
\hline$(0,0,0,1)$ & 1 \\
\hline$(0,1,0,0)$ & 1 \\
\hline$(1,0,0,0)$ & 1 \\
\hline$(0,0,1,0)$ & 1 \\
\hline$(0,0,1,1)$ & $y+y^{-1}$ \\
\hline$(0,1,1,0)$ & $y+y^{-1}$ \\
\hline$(0,0,1,2)$ & 1 \\
\hline$(0,0,2,1)$ & 1 \\
\hline$(0,1,2,0)$ & 1 \\
\hline$(0,2,1,0)$ & 1 \\
\hline$(0,1,1,1)$ & $y^{2}+2+y^{-2}$ \\
\hline$(0,1,1,2)$ & $y+y^{-1}$ \\
\hline$(0,2,1,1)$ & $y+y^{-1}$ \\
\hline$(0,1,2,1)$ & $y^{3}+y+y^{-1}+y^{-3}$ \\
\hline$(0,0,2,3)$ & 1 \\
\hline$(0,0,3,2)$ & 1 \\
\hline$(0,1,2,2)$ & $y^{4}+2 y^{2}+4+2 y^{-2}+y^{-4}$ \\
\hline$(0,2,2,1)$ & $y^{4}+2 y^{2}+4+2 y^{-2}+y^{-4}$ \\
\hline$(0,2,3,0)$ & 1 \\
\hline$(0,3,2,0)$ & 1 \\
\hline$(1,1,2,1)$ & $y^{2}+2+y^{-2}$ \\
\hline$(0,2,1,2)$ & 1 \\
\hline$(0,1,2,3)$ & $y^{3}+y+y^{-1}+y^{-3}$ \\
\hline$(0,1,3,2)$ & $y^{5}+2 y^{3}+4 y+4 y^{-1}+2 y^{-3}+y^{-5}$ \\
\hline$(0,2,3,1)$ & $y^{5}+2 y^{3}+4 y+4 y^{-1}+2 y^{-3}+y^{-5}$ \\
\hline$(0,3,2,1)$ & $y^{3}+y+y^{-1}+y^{-3}$ \\
\hline$(1,1,2,2)$ & $y+y^{-1}$ \\
\hline$(1,2,2,1)$ & $y+y^{-1}$ \\
\hline$(0,2,2,2)$ & $y^{5}+2 y^{3}+4 y+4 y^{-1}+2 y^{-3}+y^{-5}$ \\
\hline$(0,0,3,4)$ & 1 \\
\hline$(0,0,4,3)$ & 1 \\
\hline$(0,1,2,4)$ & 1 \\
\hline$(0,1,3,3)$ & $y^{6}+2 y^{4}+4 y^{2}+6+4 y^{-2}+2 y^{-4}+y^{-6}$ \\
\hline$(0,1,4,2)$ & $y^{4}+y^{2}+2+y^{-2}+y^{-4}$ \\
\hline$(0,2,2,3)$ & $y^{4}+2 y^{2}+4+2 y^{-2}+y^{-4}$ \\
\hline$(0,2,3,2)$ & $y^{8}+2 y^{6}+4 y^{4}+4 y^{2}+5+4 y^{-2}+4 y^{-4}+2 y^{-6}+y^{-8}$ \\
\hline$(0,2,4,1)$ & $y^{4}+y^{2}+2+y^{-2}+y^{-4}$ \\
\hline$(0,3,2,2)$ & $y^{4}+2 y^{2}+4+2 y^{-2}+y^{-4}$ \\
\hline$(0,3,3,1)$ & $y^{6}+2 y^{4}+4 y^{2}+6+4 y^{-2}+2 y^{-4}+y^{-6}$ \\
\hline$(0,3,4,0)$ & 1 \\
\hline$(0,4,2,1)$ & 1 \\
\hline$(0,4,3,0)$ & 1 \\
\hline$(1,1,3,2)$ & $y^{2}+2+y^{-2}$ \\
\hline$(1,2,2,2)$ & $y^{4}+3 y^{2}+6+3 y^{-2}+y^{-4}$ \\
\hline$(1,2,3,1)$ & $y^{2}+2+y^{-2}$ \\
\hline
\end{tabular}

TABLE I. Protected Spin Characters at a fiber-base symmetric point.

closely analogous to those studied in [16], Sec. 5.3 and [15], Sec. 7.2. Let us comment on how the expression $\mathbb{U}$ obtained here compares with these works.

The difference with [15], Sec. 7.2 is the factor $\mathbb{U}\left(\mathbb{R}^{+}\right)$, where we find additional infinite towers of states $\Omega\left(\gamma_{1}+\right.$ $\left.\gamma_{2}+k \gamma_{D 0} ; y\right)=\Omega\left(\gamma_{3}+\gamma_{4}+k \gamma_{D 0} ; y\right)=y+y^{-1}$ for all 
$k \geq 0$, as well as additional states $\Omega\left(n \gamma_{D 0} ; y\right)=y^{-1}(1+$ $\left.y^{2}\right)^{2}$ for all $n \geq 1$ directly in Fig. 1. Here $\gamma_{D 0}$ is the charge of a $D 0$-brane, while $\gamma_{1}+\gamma_{2}$ is the charge of $D 2_{f}$ and $\gamma_{3}+$ $\gamma_{4}$ the charge of $D 0-\overline{D 2}_{f}$. A resolution of this discrepancy is that Closset and Del Zotto [15] studied stable quiver representations, while our results indicate that $\mathbb{U}$ includes contributions from threshold states such as $D 2-D 0$. These additional states can be expected correspond to KaluzaKlein modes of an $M 2$-brane wrapping the fiber $\mathbb{P}^{1}$ [27], and can also be seen by studying the $\mathbb{Z}_{4}$ symmetry of $\mathbb{U}$ inherited from the BPS quiver [11]. Pure $D 0$ states are also expected from previous works, e.g., [7,16,28]. However, since networks only compute the unrefined index $\Omega\left(n \gamma_{D 0}\right)=-4$, we adopted the motivic refinement $\Omega\left(n \gamma_{D 0} ; y\right)=y^{-1}\left(1+y^{2}\right)^{2}$ from the recent work [7]. (The counting of $D 0$ branes of [7], which agrees with exponential networks in the unrefined limit, differs from the physical counting of [28]. The relation between the two has been discussed in [7].)

Comparing with [16] we find direct agreement for the BPS spectrum at a fiber-base symmetric point, recovering and extending their results in the limit $y \rightarrow-1$. At generic $y$, our results agree with predictions from the Coulomb branch and attractor flow formulas of [29,30], computed with [31]. By extension our results should also agree with recent computations [32], confirming earlier predictions of [33].

After this Letter appeared, we were informed that Mozgovoy and Pioline [7] obtained an exact expression for the generating function of unframed stacky invariants of the BPS quiver associated to the model we study. This, together with related conjectures on attractor invariants, would provide an alternative derivation of (2).

Interesting applications include a relation to the 5D superconformal index [34], as well as comparing with computations of Vafa-Witten invariants with different techniques [35-37].

I would like to thank Sibasish Banerjee and Mauricio Romo for collaboration on related projects and Fabrizio del Monte, Michele Del Zotto, and Boris Pioline for correspondence. This work is supported by NCCR SwissMAP, funded by the Swiss National Science Foundation.

*longhip@phys.ethz.ch

[1] N. Seiberg and E. Witten, Nucl. Phys. B426, 19 (1994); B430, 485(E) (1994).

[2] N. Seiberg, Phys. Lett. B 388, 753 (1996).

[3] M. Kontsevich and Y. Soibelman, arxiv:0811.2435.
[4] D. R. Morrison and N. Seiberg, Nucl. Phys. B483, 229 (1997).

[5] M. R. Douglas, S. H. Katz, and C. Vafa, Nucl. Phys. B497, 155 (1997).

[6] P. Bousseau, arXiv:1909.02985.

[7] S. Mozgovoy and B. Pioline, arXiv:2012.14358.

[8] D. Joyce and Y. Song, arXiv:0810.5645.

[9] D. Gaiotto, G. W. Moore, and A. Neitzke, Commun. Math. Phys. 299, 163 (2010).

[10] D. Gaiotto, G. W. Moore, and A. Neitzke, Adv. Math. 234, 239 (2013).

[11] P. Longhi, Ann. Henri Poincare 19, 775 (2018).

[12] M. Gabella, P. Longhi, C. Y. Park, and M. Yamazaki, J. High Energy Phys. 07 (2017) 032.

[13] D. Gaiotto, G. W. Moore, and A. Neitzke, Ann. Henri Poincare 14, 1643 (2013).

[14] R. Eager, S. A. Selmani, and J. Walcher, J. High Energy Phys. 08 (2017) 063.

[15] C. Closset and M. Del Zotto, arXiv:1912.13502v2.

[16] S. Banerjee, P. Longhi, and M. Romo, arXiv:2012.09769.

[17] D. Gaiotto, G. W. Moore, and A. Neitzke, Adv. Theor. Math. Phys. 17, 241 (2013).

[18] M. Del Zotto and A. Sen, Commun. Math. Phys. 357, 1113 (2018).

[19] W.-y. Chuang, D.-E. Diaconescu, J. Manschot, G. W. Moore, and Y. Soibelman, Adv. Theor. Math. Phys. 18, 1063 (2014).

[20] M. R. Douglas, B. Fiol, and C. Romelsberger, J. High Energy Phys. 09 (2005) 057.

[21] T. Bridgeland, Ann. Math. 166, 317 (2007).

[22] A. Hanany and A. Iqbal, J. High Energy Phys. 04 (2002) 009.

[23] M. Alim, S. Cecotti, C. Cordova, S. Espahbodi, A. Rastogi, and C. Vafa, Commun. Math. Phys. 323, 1185 (2013); Adv. Theor. Math. Phys. 18, 27 (2014).

[24] S. Banerjee, P. Longhi, and M. Romo, Ann. Henri Poincare 20, 4055 (2019).

[25] S. Banerjee, P. Longhi, and M. Romo, arXiv:1910.05296.

[26] D. Galakhov, P. Longhi, and G. W. Moore, Commun. Math. Phys. 340, 171 (2015).

[27] R. Gopakumar and C. Vafa, arXiv:9809187; arXiv:9812127.

[28] Z. Duan, D. Ghim, and P. Yi, arxiv:2011.04661.

[29] J. Manschot, B. Pioline, and A. Sen, J. High Energy Phys. 07 (2011) 059; 11 (2012) 023; 05 (2011) 057; 05 (2013) 166.

[30] S. Alexandrov and B. Pioline, Adv. Theor. Math. Phys. 23, 627 (2019).

[31] B. Pioline, Mathematica package "CoulombHiggs", https:// www.lpthe.jussieu.fr/ pioline/computing.html.

[32] G. Beaujard, J. Manschot, and B. Pioline, arXiv:2004.14466.

[33] J. Manschot, Lett. Math. Phys. 103, 895 (2013).

[34] A. Iqbal and C. Vafa, Phys. Rev. D 90, 105031 (2014).

[35] K. Yoshioka, Math. Ann. 302, 519 (1995).

[36] F. Denef and G. W. Moore, J. High Energy Phys. 11 (2011) 129.

[37] S. Alexandrov, arXiv:2005.03680; arXiv:2006.10074. 